\title{
Comprehensive analysis reveals GRP94 is associated with worse prognosis of breast cancer
}

\author{
Ting Wang ${ }^{1 \#}$, Lei Yang ${ }^{2 \#}$, Chunxiao $\mathrm{Li}^{1}$, Jinsong Wang ${ }^{1}$, Jingyao Zhang ${ }^{1}$, Yantong Zhou ${ }^{1}$, Fangzhou Sun ${ }^{1}$, \\ Haijuan Wang ${ }^{1}$, Fei Ma ${ }^{3}$, Haili Qian ${ }^{1 \wedge}$
}

${ }^{1}$ State Key Laboratory of Molecular Oncology, National Cancer Center/National Clinical Research Center for Cancer/Cancer Hospital, Chinese Academy of Medical Sciences and Peking Union Medical College, Beijing, China; ${ }^{2}$ Department of Imaging Diagnostic, National Cancer Center/ National Clinical Research Center for Cancer/Cancer Hospital, Chinese Academy of Medical Sciences and Peking Union Medical College, Beijing, China; ${ }^{3}$ Department of Medical Oncology, National Cancer Center/National Clinical Research Center for Cancer/Cancer Hospital, Chinese Academy of Medical Sciences and Peking Union Medical College, Beijing, China

Contributions: (I) Conception and design: H Qian, L Yang, T Wang; (II) Administrative support: H Qian, F Ma; (III) Provision of study materials or patients: H Wang, C Li; (IV) Collection and assembly of data: T Wang, J Wang, F Sun; (V) Data analysis and interpretation: J Zhang, Y Zhou; (VI) Manuscript writing: All authors; (VII) Final approval of manuscript: All authors.

\#These authors contributed equally to the work.

Correspondence to: Haili Qian. State Key Laboratory of Molecular Oncology, National Cancer Center/National Clinical Research Center for Cancer/ Cancer Hospital, Chinese Academy of Medical Sciences and Peking Union Medical College, No. 17 Pan-jia-yuan South Lane, Chaoyang District, 100021, Beijing, China. Email: qianhaili001@163.com.

Background: Breast cancer (BC) is the most common cancer diagnosed in women around the world. Glucose-related protein 94 (GRP94) is a molecular chaperone on the endoplasmic reticulum (ER) that is associated with many malignancies, although its role in breast carcinogenesis has remained unclear. This study aimed to investigate the expression of GRP94 in BC and its relationship with BC clinicopathological features and prognosis based on a comprehensive analysis.

Methods: The mutation and expression patterns of GRP94 in multiple cancers were elucidated from TCGA data. A GRP94 IS (immune score) was generated from breast tumors in Chinese women by multiplying the staining intensity and the percentage of positive cells. The relationship between GRP94 expression and clinicopathological parameters in TMA samples was identified by Spearman correlation analysis. We established a GRP94 co-expression interaction network from two databases (TCGA and STRING). Overall survival (OS) and relapse-free survival (RFS) were determined via the KM-plotter analysis platform.

Results: GRP94 is mutated in most cancer types, and the average mutation frequency is 1.1\%. GRP94 expression in $\mathrm{BC}$ was in the middle of the expression levels of the analyzed cancer types. The protein level of GRP94 was significantly higher in BC tissues than in normal breast tissues. A high level of GRP94 was positively associated with the levels of PR and AR and negatively associated with the level of EGFR but was not associated with age, pathological types, pathological grades, clinical stages or the levels of ER, HER2, P53, Ki67, or CK5/6. High expression of GRP94 predicted decreased OS and RFS in BC. The cluster analysis of the GRP94 gene coexpression network showed six dominant biological events, including ribosome biogenesis, amino acid activation, ER stress, protein folding and protein localization to the nucleus, cell cycle processes and ubiquitin-protein ligase activity involved in the mitotic cell cycle.

Conclusions: The study suggests that GRP94 could be a potential prognostic factor in BC.

Keywords: Breast cancer (BC); bioinformatics analysis; glucose-related protein 94 (GRP94); prognosis

Submitted Apr 16, 2020. Accepted for publication Sep 12, 2020.

doi: $10.21037 /$ tcr-20-1853

View this article at: http://dx.doi.org/10.21037/tcr-20-1853

^ ORCID: 0000-0003-0652-4801. 


\section{Introduction}

Breast cancer (BC) is one of the most common types of female cancer worldwide, threatening women's health and lives. There were 248,620 new BC cases in females, corresponding to an age-standardized incidence rate of 29 per 100,000 Chinese women, which compares with approximately 120 per 100,000 in westernized population (1). However, the recent age-standardized data (in patients from 0 to 74 years old) show that the 5 -year relative survival varied from $58 \%$ to $90 \%$, with a median survival of $88 \%$, approximately $11 \%$ of worldwide $\mathrm{BC}$ cases occur in China (2). Therefore, it is necessary to find specific biomarkers in $\mathrm{BC}$ and explore the related mechanisms. Glucose-regulated protein 94 (GRP94) (3), also named HSP90B1 or GP96, a member of the adenosine triphosphate (ATP)-metabolizing family, is a molecule chaperone that can stabilize and fold other proteins in the endoplasmic reticulum (ER). GRP94 has been shown to be an essential master chaperone for multiple receptors, participating in activities including protein folding, ER quality control, and calcium homeostasis (4). Clinically, the overexpression of GRP94 has been reported in multiple types of malignances, such as esophageal cancer, hepatocellular carcinoma (5), non-small cell lung cancer $(6,7)$, colorectal carcinoma (8), gastric cancer (9), and esophageal adenocarcinoma (10). GRP94 contributes to the development of an aggressive phenotype in BC cells, including their invasive capacity and sensi to doxorubicin (11). However, the difference in GRP94 expression between $\mathrm{BC}$ tissue and normal breast tissue is not well understood, and whether GRP94 expression is clinically indicative of $\mathrm{BC}$ remains unclear.

In this study, we validated the expression pattern of GRP94 in BC and investigated the prognostic roles of GRP94 in BC patients. Then, we established a GRP94 gene coexpression interaction network to explore its potential mechanism in BC. Our study provides evidence that GRP94 could be a prognostic factor and potential therapeutic target in $\mathrm{BC}$.

\section{Methods}

\section{Tissue microarrays (TMAs)}

The BC TMAs, which were consisted of primary invasive breast carcinomas $(n=160)$ from Chinese women who underwent surgical resection between 2001 and 2014, were purchased from Shanghai Outdo Biotech Co., Ltd. The TMAs were constructed with $1.5 \mathrm{~mm}$ cores from formalinfixed paraffin-embedded tissue blocks, and 10 normal breast tissue cores were also included at the end of the TMAs, among which there was a loss of $7 \mathrm{BC}$ cases and 4 normal breast tissues because of immunohistochemistry detachment. Patient age, pathologic diagnosis, tumor size, lymph node status, presence of other metastasis, and histological grade were determined by pathology reports and medical records. The overall survival (OS) was defined as the time from the date of surgery to the date of death. Patients were staged using the American Joint Committee on Cancer $6^{\text {th }}$ Edition guidelines.

\section{Immunobistochemistry}

GRP94 expression was assessed by immunohistochemistry using a rat monoclonal anti-GRP94 antibody (Abcam, UK). Immunohistochemical staining was performed as previously described (12). In brief, the sections were deparaffinized with xylenes and rehydrated with an ethanol gradient. Then, the sections were submerged into sodium citrate buffer, microwaved for $17 \mathrm{~min}$ and then cooled at room temperature for $90 \mathrm{~min}$. The sections were treated with $3 \%$ hydrogen peroxide and then incubated with goat serum to block nonspecific binding for $20 \mathrm{~min}$. The anti-GRP94 antibody was incubated with sections overnight at $4{ }^{\circ} \mathrm{C}$ at a 1:100 dilution. After washing, the sections were incubated with a secondary antibody (Zhongshan Biotechnology Company, China) for $60 \mathrm{~min}$, followed by further staining with diaminobenzidine for visualization. The IS was generated by multiplying the cytoplasmic staining intensity ( weak $=1$, moderate $=2$, strong $=3$ ) and the percent of positive cells $(<5 \%=0,5-30 \%=1,30-50 \%=2,50-80 \%$ $=3$, and $>80 \%=4$ ) and was assessed by one pathologist. The cohort of 160 samples was divided into high- and lowGRP94 expression groups based on a cutoff score of 6 .

\section{Bioinformatic analysis}

The mutation and expression patterns of GRP94 from 26 cancer types were obtained from the cBioPortal analysis platform (http://www.cbioportal.org/), which include Stomach Adenocarcinoma [440], Sarcoma [255], Ovarian Cancer [585], Bladder Urothelial Carcinoma [411], Liver Hepatocellular Carcinoma [372], Uterine Corpus Endometrial Carcinoma (Uterine CS, 529), 
Esophageal Carcinoma [559], Prostate Adenocarcinoma [494], Skin Cutaneous Melanoma [448], Mesothelioma [87], Head and Neck Squamous Cell Carcinoma [523], Lung Adenocarcinoma [566], Brain Lower Grade Glioma [514], Kidney Renal Papillary Cell Carcinoma (pRCC, 283), Breast Invasive Carcinoma [1,084], Lung Squamous Cell Carcinoma [487], Kidney Renal Clear Cell Carcinoma (ccRCC, 512), Pancreatic Adenocarcinoma [184], Glioblastoma Multiforme (GBM, 592), Colorectal Adenocarcinoma [594], Cholangiocarcinoma [36], Acute Myeloid Leukemia (AML, 200), Pheochromocytoma and Paraganglioma (PCPG, 178), Thymoma [123], Uveal Melanoma [80]. The expression of GRP94 in a TMA including 165 cases was obtained from the ONCOMINE database (Curtis Breast TMA, https://www.oncomine.org/ resource/login.html), which included 144 normal tissues and 21 invasive breast carcinomas. A gene was considered to be coexpressed with GRP94 when the absolute value of the Spearman correlation coefficient between its expression and that of GRP94 was equal to or greater than 0.35. STRING was used to generate the GRP94 gene coexpression interaction network (https://string-db.org). The DAVID analysis platform was used for the functional cluster analysis of the genes (https://david.ncifcrf.gov/).

\section{Survival analysis}

The Kaplan Meier plotter database (www.kmplot.com), was applied to evaluate the relationship between GRP94 and clinical prognosis(ref) in BC. And 1402 BC cases included in this database were used to analysis the OS of $\mathrm{BC}$ patients, and 3915 BC cases were used to analysis the relapse free survival (RFS). And we split the patients by using best performing expression value of GRP94 as cutoff into two groups (high vs. low). We evaluated the survival time of BC patients using a Kaplan-Meier survival plot. The hazard ratio (HR) with $95 \%$ confidence intervals and $\log$ rank $\mathrm{P}$ value were calculated and displayed on the plot.

\section{Statistical analysis}

The $\chi^{2}$ test was used to examine the expression difference between normal breast tissues and $\mathrm{BC}$ tissues. The spearman's rank correlation coefficients between expression of GRP94 and clinical pathological features were analyzed and examined by IBM SPSS Statistics 22 . The pictures were made using Adobe Photoshop CS6. The survival analysis was carried out using GraphPad Prism 7.

\section{Statement of ethics approval}

Part of our study was investigated using public databases including Oncomine, cBioPortal and Kaplan Meier plotter databases. The BC TMAs were purchased from Shanghai Outdo Biotech Co., Ltd. All procedures performed in studies were in accordance with the declaration of Helsinki (as revised in 2013). This study was approved by the Ethics Committee of Cancer Institute and Hospital, Chinese Academy of Medical Sciences (Approval No.16-038/1117), and all of the participants had been giving informed consent before the study.

\section{Results}

\section{Mutation and expression of GRP94 in various cancers}

We studied the mutation and expression of GRP94 in 26 cancers, including data from 10,136 patients, using the cBioPortal database analysis platform and found that GRP94 was mutated in most cancer types (except for AML, cholangiocarcinoma and chronic lymphocytic leukemia) and that the average mutation frequency was $1.1 \%$. Gene amplification, deletion and truncation and missense mutations were the main types of alterations, and different cancers had different frequencies of each type. Point mutations in GRP94 mainly existed in stomach cancer, uterine cancer and liver cancer, and gene amplification was more common in sarcoma, ovarian cancer, bladder cancer and prostate cancer than in other cancer types (Figure 1A). The mutation frequency of GRP94 in BC was relatively low, and thus, it is speculated that the effect of GRP94 on malignant BC may be carried out at the transcriptome level. According to the TCGA database, GRP94 is expressed in multiple cancers, and its expression is relatively low in glioma and high in liver cancer and thyroid cancer. GRP94 is also expressed in BC (Figure 1B).

\section{Expression of GRP94 in BC}

GRP94 expression was first verified through the Curtis Breast TMA in the ONCOMINE database (13). Consistent with the earlier observation in NSCLC (6), the expression level of GRP94 in BC was significantly higher in invasive breast carcinoma than in normal breast tissues $(\mathrm{P}<0.01$, Figure 2). To confirm this result, we detected GRP94 expression in a BC TMA containing 160 cases by immunochemistry. The results demonstrated that GRP94 was mainly expressed in both the cytoplasm and nucleus. 
A
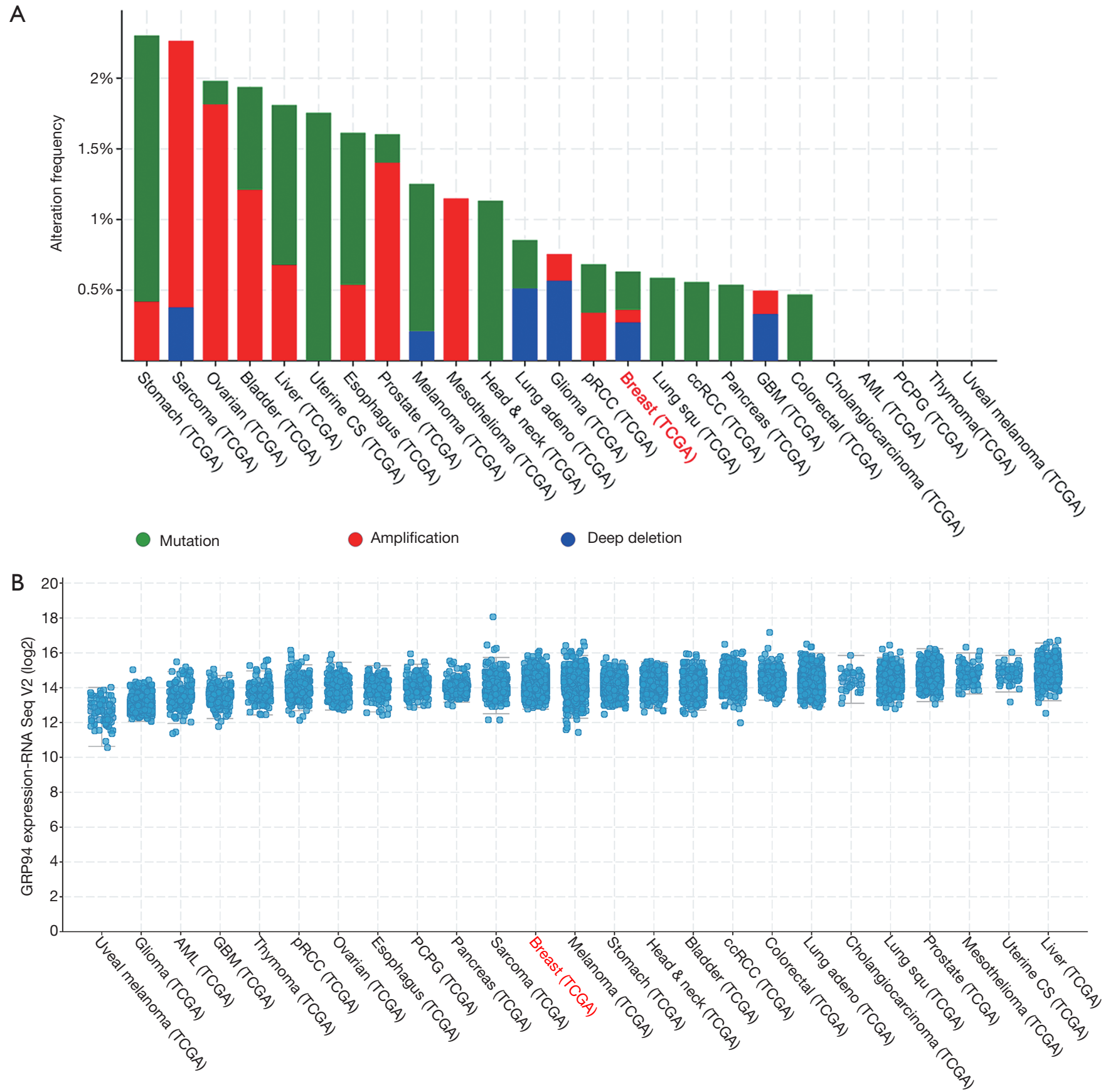

Figure 1 Genetic alteration and expression of GRP94 in multiple cancers according to data from the cBioportal platform. (A) The mutation frequency of GRP94 among multiple cancers; (B) the expression of GRP94 among multiple cancers. GRP94, glucose related protein 94.

According to the immunohistochemistry scores, the expression of GRP94 was significantly higher in BC tissues than in normal breast tissues $(\mathrm{P}<0.05$, Figure $3 A, B)$. In the 160 cases contained within the TMA, the rate of positive
GRP94 expression was $99.346 \%(152 / 153)$ in BC, and there were 113 cases with high expression of GRP94, 40 cases with low expression according to the cutoff IS criterion of 6. These data indicate that GRP94 is associated with the 


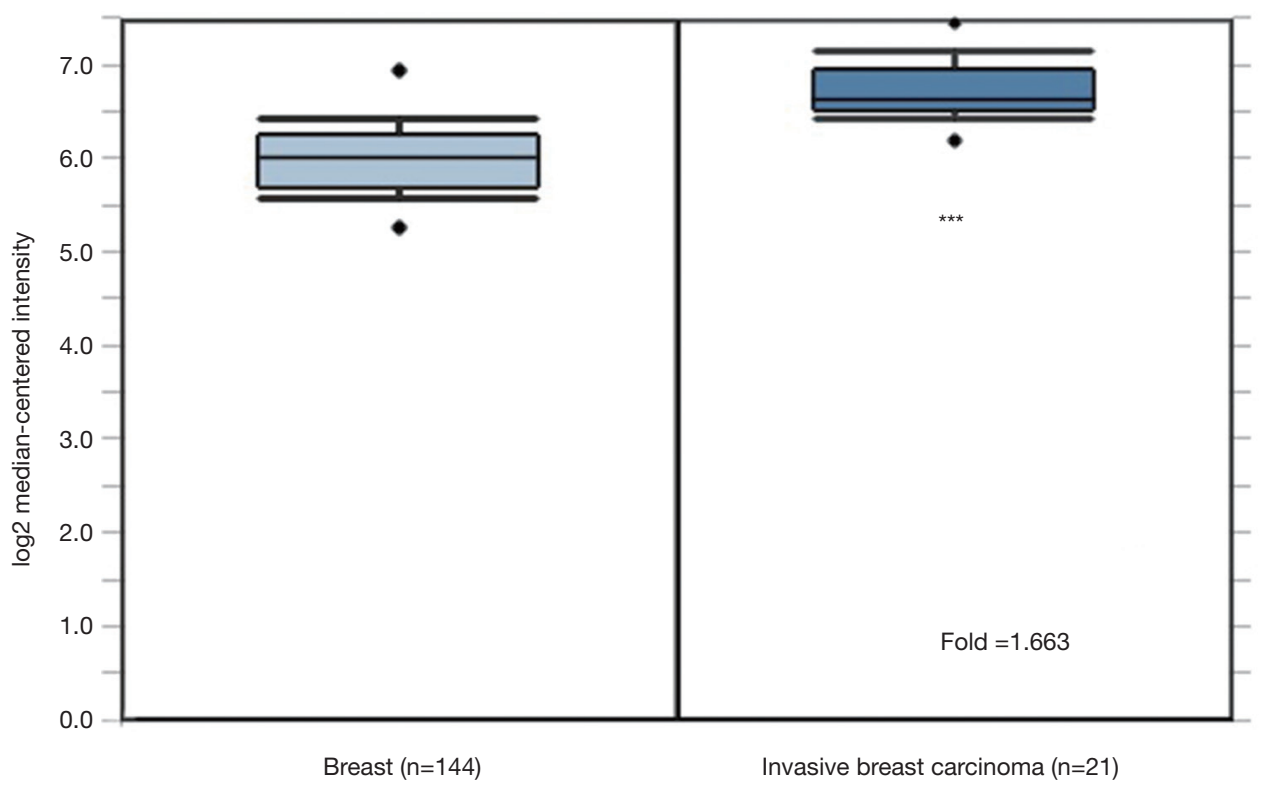

Figure 2 Expression of GRP94 in 165 BC patients from the ONCOMINE database. ***, $\mathrm{P}<0.001$. BC, breast cancer.
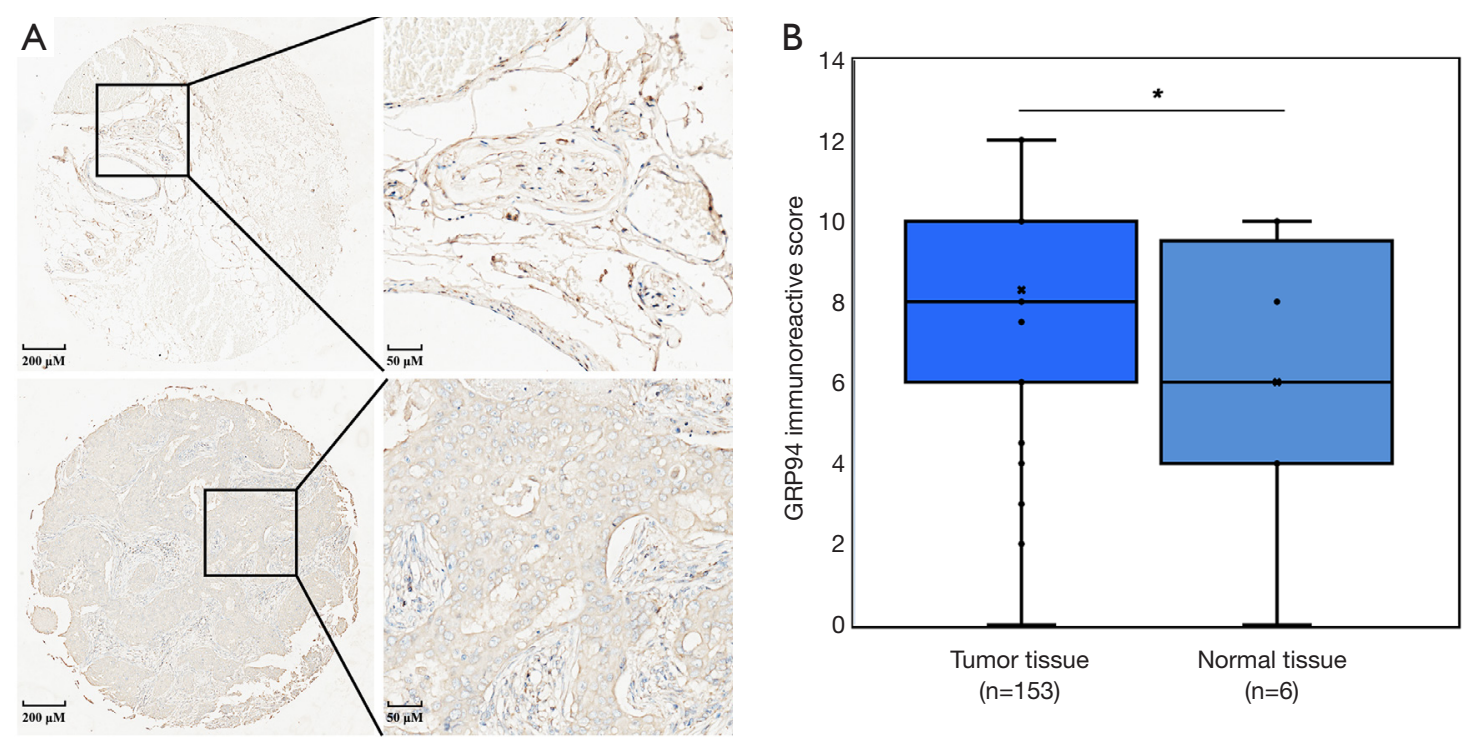

Figure 3 Expression of GRP94 in the TMA. (A) Top: normal breast tissue, IS =4; bottom: BC tissue, IS =12; (B) Box plot of the immunoreactive score of GRP94 in tumor tissue and normal tissue. * $\mathrm{P}<0.05$. TMA, tissue microarray; IS, immune score.

development of BC.

\section{Relationship between GRP94 and clinical pathological features}

Based on the immunochemistry results, the association between GRP94 expression and clinicopathological characteristics was further assessed. The results showed that high GRP94 expression positively correlated with higher $\mathrm{PR}$ and $\mathrm{AR}$ expression $(\mathrm{P}=0.0294, \mathrm{P}=0.0052)$ and correlated with lower EGFR expression ( $\mathrm{P}=0.0122)$. GRP94 expression had no such correlation with ER, HER2, P53, Ki67, or CK5/6 levels, age, clinical stage, tumor grade, lymph node status, or histologic type (Tables 1,2). These results suggest 
Table 1 Correlation between expression of GRP94 and clinicopathological parameters

\begin{tabular}{|c|c|c|c|c|}
\hline Characteristic & \multicolumn{2}{|c|}{ GRP94 } & $r_{\mathrm{s}}$ & $P$ value \\
\hline \multicolumn{5}{|l|}{ Age (years) } \\
\hline$<55$ & 65 (42.5\%) & 27 (17.6\%) & 0.08117 & 0.3091 \\
\hline$\geq 55$ & $48(31.4 \%)$ & $13(8.5 \%)$ & & \\
\hline I & $11(7.2 \%)$ & $1(0.6 \%)$ & -0.04894 & 0.5467 \\
\hline II & $68(44.4 \%)$ & $26(17.0 \%)$ & & \\
\hline III & 34 (22.2\%) & 12 (7.8\%) & & \\
\hline \multicolumn{5}{|l|}{ ER } \\
\hline \multicolumn{5}{|l|}{ PR } \\
\hline Pos & $66(43.1 \%)$ & $5(3.3 \%)$ & 0.1728 & $0.0294^{*}$ \\
\hline $\mathrm{Neg}$ & $46(30.1 \%)$ & 13 (8.5\%) & & \\
\hline NA & $1(0.6 \%)$ & $3(1.9 \%)$ & & \\
\hline \multicolumn{5}{|l|}{ AR } \\
\hline Pos & $99(64.7 \%)$ & 34 (22.2\%) & 0.2207 & $0.0052^{\star \star}$ \\
\hline Neg & $14(9.1 \%)$ & $6(3.9 \%)$ & & \\
\hline \multicolumn{5}{|l|}{ EGFR } \\
\hline \multicolumn{5}{|l|}{ P53 } \\
\hline \multicolumn{5}{|l|}{ HER2 } \\
\hline Pos & 39 (25.5\%) & $12(7.8 \%)$ & 0.1079 & 0.1758 \\
\hline Neg & 74 (48.4\%) & 27 (17.6\%) & & \\
\hline NA & $0(0.0 \%)$ & $1(0.6 \%)$ & & \\
\hline \multicolumn{5}{|l|}{ Ki67 } \\
\hline Pos & $112(73.2 \%)$ & $36(23.5 \%)$ & 0.1332 & 0.0941 \\
\hline Neg & $1(0.6 \%)$ & $4(2.6 \%)$ & & \\
\hline \multicolumn{5}{|l|}{ Tumor grade } \\
\hline I & $32(20.9 \%)$ & $16(10.5 \%)$ & 0.1439 & 0.0704 \\
\hline II & 79 (51.6\%) & 22 (14.4\%) & & \\
\hline III & $2(1.3 \%)$ & $2(1.3 \%)$ & & \\
\hline
\end{tabular}

ER, estrogen receptor; PR, progesterone receptor; AR, androgen receptor; EGFR, epidermal growth factor receptor; Pos, positive; Neg, negative; NA, not available. *, $\mathrm{P}<0.05,{ }^{*}, \mathrm{P}<0.01$. 
Table 2 Correlation between expression of GRP94 and lymph node status and histologic type

\begin{tabular}{lccc}
\hline \multirow{2}{*}{ Characteristic } & \multicolumn{2}{c}{ GRP94 } & $\chi^{2}$ \\
\cline { 2 - 4 } LN status & Ligh $(\mathrm{n}=113)$ & $21(13.7 \%)$ & 0.3625 \\
Pos & $69(45.1 \%)$ & $18(11.8 \%)$ & 0.5471 \\
Neg & $39(25.5 \%)$ & $1(0.6 \%)$ & \\
NA & $5(3.3 \%)$ & & \\
Histologic type & & $35(22.9 \%)$ & 1.9633 \\
IDC & $110(71.9 \%)$ & $5(3.3 \%)$ & \\
ILC & $3(1.9 \%)$ & & \\
\hline
\end{tabular}

LN, lymph node; IDC, invasive ductal carcinoma; ILC, invasive lobular carcinoma; Pos, positive; Neg, negative; NA, not available.

that GRP94 may play a role in hormone receptor-positive cancer biology and inhibition of cancer cell proliferation.

\section{Relationship between GRP94 and clinical prognosis}

After identifying the relationship between GRP94 and the clinicopathological parameters of $\mathrm{BC}$, the relationship of GRP94 with the prognosis of BC was demonstrated. According to the prognostic information associated with the TMA BC samples, we found that patients with high GRP94 expression had a lower OS than those with low GRP94 expression (Figure 4A), but the result was not statistically significant $(\mathrm{P}=0.1022)$, which may be due to an insufficient sample size. Therefore, we further analyzed the results in the KM-plotter dataset (14), and the results showed that patients with high expression of GRP94 had decreased OS and RFS $\left(\mathrm{P}=0.042, \mathrm{P}=7.6 \times 10^{-14}\right.$ ) (Figure $4 B, C$ ).

\section{GRP94 gene coexpression bioinformatic analysis}

Based on the relationship between GRP94 and the prognosis of BC patients, we aimed to explore the potential mechanism by which GRP94 promotes the development of BC. A previous report indicated that GRP94 chaperones several proteins essential in carcinogenesis. According to the transcriptome data of BC in TCGA, we obtained a cluster of genes that were highly correlated with GRP94 expression patterns in BC patients. These genes may be involved in certain biological processes in BC cells along with GRP94. Then, a GRP94 coexpression interaction network was generated using the STRING database (Figure 5). The interaction network showed five dominant biological events, including ribosome biogenesis, amino acid activation,
ER stress, protein folding and protein localization to the nucleus, cell cycle processes and ubiquitin-protein ligase activity involved in the mitotic cell cycle. This analysis suggests that, in addition to its effects on protein folding and ER stress, GRP94 may also promote poor prognosis in $\mathrm{BC}$ by affecting ribosomal protein production and the cell cycle processes of BC cells.

\section{Discussion}

$\mathrm{BC}$ is the most common female cancer diagnosed worldwide. It is also the leading cause of female cancer death, yet its potential pathogenesis is still not comprehensively understood. In China, due to the huge population base, $11.2 \%$ of $\mathrm{BC}$ cases diagnosed worldwide occurred in China in 2012 (2). The number of deaths related to $\mathrm{BC}$ is increasing, although the incidence rate is low (1); and thus, even a tiny increase will cause a considerable loss of life. Therefore, more biomarkers are needed to precisely predict $\mathrm{BC}$ progression and prognosis.

GRP94, a member of the heat shock protein family, is the major calcium-binding protein in the ER and has specific protein clients (15). When it is under ER stress, which means low glucose and hypoxic conditions, GRP94 will be activated to fold and assemble proteins to support cell survival (16). High expression of GRP94 has been reported to correlate with advanced stage and poor survival in multiple carcinomas, such as head and neck cancer, nonsmall-cell lung cancer (6), gallbladder cancer (17), colorectal cancer (8), and esophageal cancer (10). Other studies revealed that GRP94 overexpression was closely linked to the malignant phenotype of cancer cells in melanoma (18), ovarian cancer (19), multiple myeloma (20), lung cancer (21), 
A

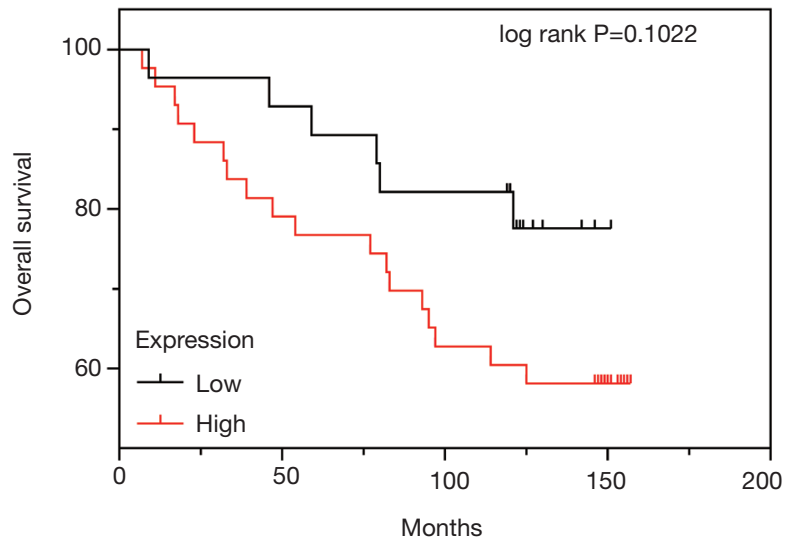

B

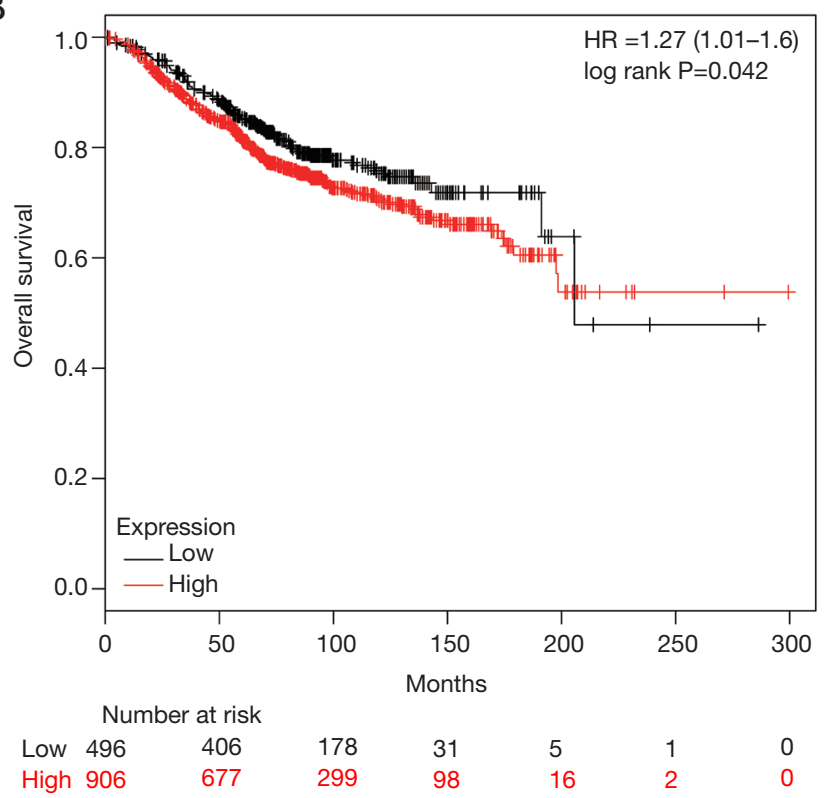

C

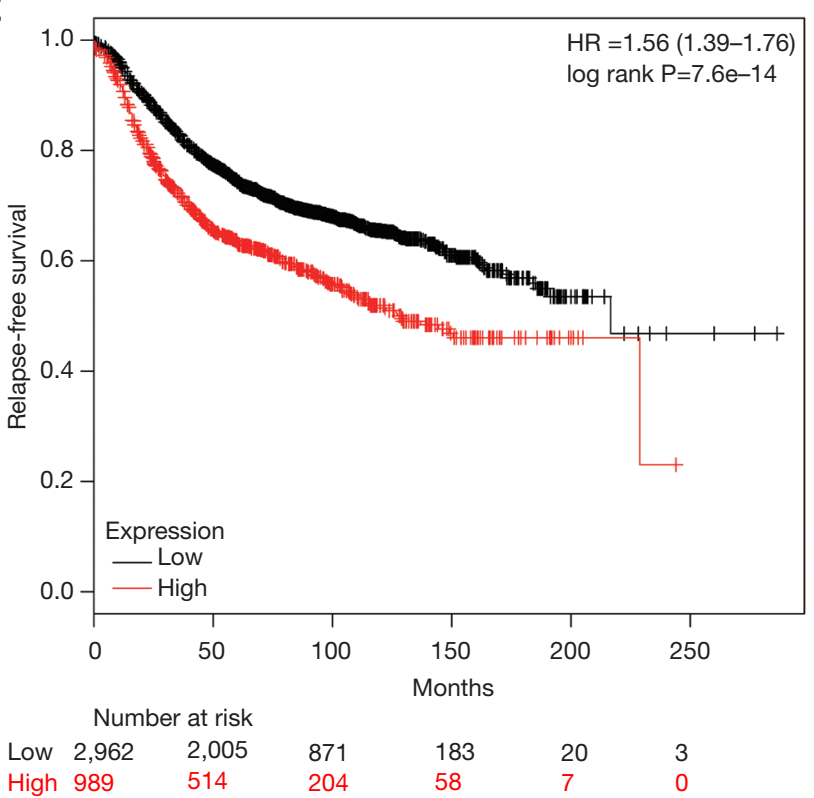

Figure 4 Overall and disease-free survival based on the KM-plotter database and GRP94 staining. (A) OS curve for the 150 cases of BC in the TMA; (B,C) OS and RFS curves of BC patients from the KM plotter dataset. KM-plotter database, Kaplan Meier plotter database; OS, overall survival; RFS, relapse-free survival.

inflammation-associated colon cancer (22), papillary thyroid cancer (23), and hepatocellular carcinoma (24) and gastric cancer cell lines (25). Consistently, from both databases and the BC TMA, our study identified that the expression of GRP94 in BC tissues was significantly higher than that in normal breast tissues, and we found that in the KMplotter database, the OS and relapse-free survival (RFS) of patients with high expression of GRP94 was significantly lower than that of patients with low expression of GRP94, suggesting that high expression of GRP94 predicts a poor prognosis in $\mathrm{BC}$.
It is universally known that triple-negative breast cancer (TNBC) is aggressive high metastatic potential and have the worst prognosis and distant metastasis-free survival among all subtypes of BC (26). Few targeted therapies are available for the patients with TNBC (27). And AR staining is common to TNBC with high expression of EGFR (28). According to our research, patients with high expression of GRP94 usually showed positive staining of AR. In the meanwhile, these patients have a higher positive rate of EGFR. Therefore, we inferred that GRP94 may play an important role in the development of TNBC 


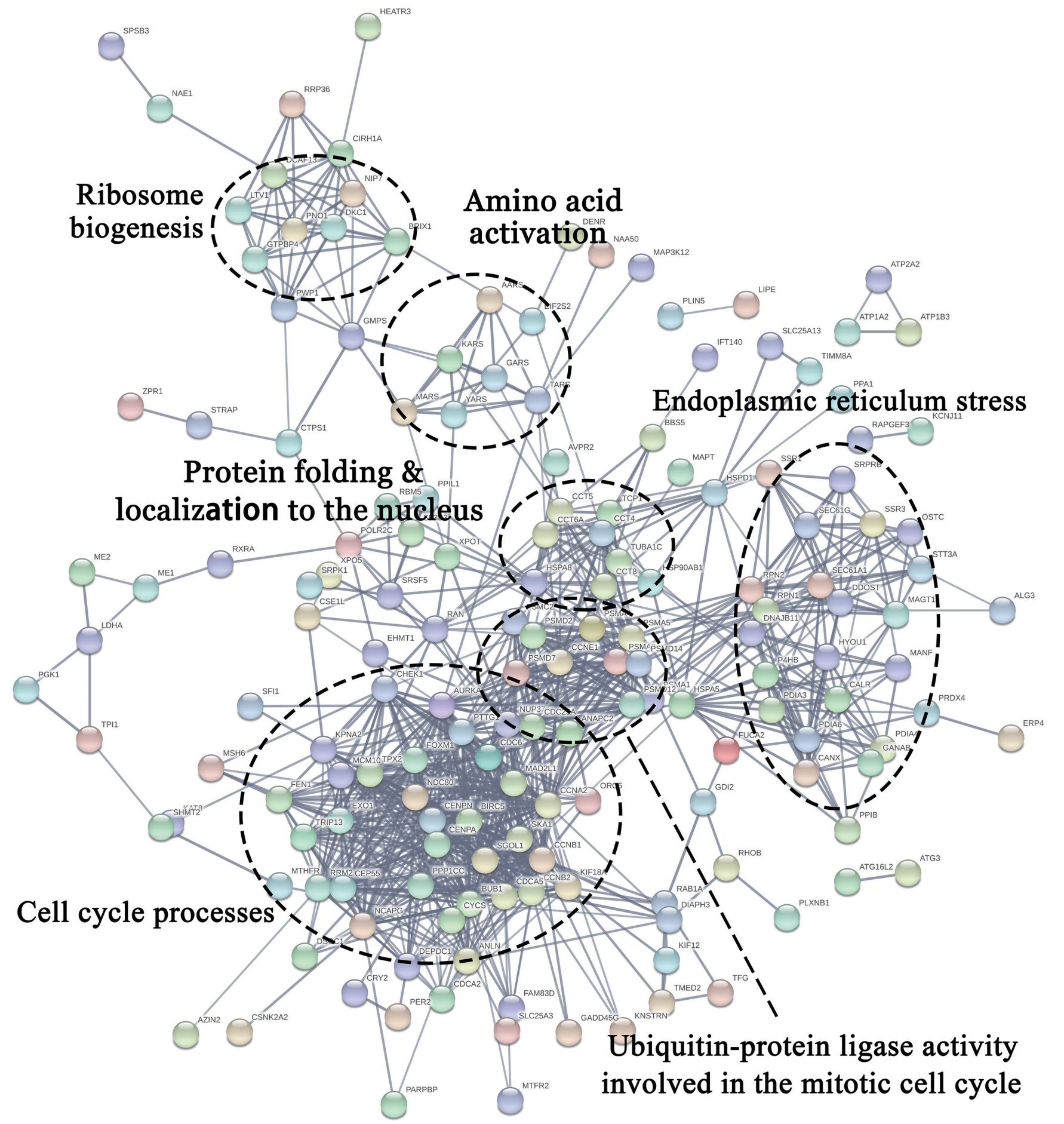

Figure 5 GRP94 gene coexpression network functional cluster analysis. Each colored dot represents a GRP94 coexpression gene. Each dotted circle represents a cluster term. 
patients with high expression of EGFR and AR staining. In human oropharyngeal carcinoma, EGFR support the radioresistance by activating ER stress signaling PERKeIF2alpha-GRP94 (29). Consequently, the high expression of GRP94 maybe associated with the metastasis and radioresistance of EGFR high expression TNBC patients. GRP94 is a potential prognosis predictor and therapeutic target in EGFR positive metastatic TNBC.

GRP94 plays a role in cellular physiological processes, including protein folding, calcium homeostasis, ER quality control and ER stress (4). The mechanism by which GRP94 promotes the development of BC may be through the above biological events. In our research, the GRP94 gene coexpression interaction network in $\mathrm{BC}$ showed five dominant biological events, including ribosome biogenesis, amino acid activation, ER stress, protein folding and localization to the nucleus, cell cycle processes and ubiquitin-protein ligase activity involved in the mitotic cell cycle. It is relatively clear that the main task of GRP94 is to support the folding and assembly of secretory and membrane proteins (15), a function that is related to processes such as ribosome biogenesis and amino acid activation. Additionally, GRP94 and GRP78 are considered characteristic molecules of the unfolded protein response (UPR), and thus, GRP94 is able to increase the UPR to promote the folding capacity of ER proteins in $\mathrm{BC}$ patients. NF- $\mathrm{KB}$ is able to increase the expression of GRP94 to promote hepatocyte cell cycle progression and transition (30). Interestingly, according to the interaction network cluster analysis, we found that GRP94 may influence the development of BC through effects on cell cycle progression.

\section{Conclusions}

Our study indicated that GRP94 could be a potential biomarker for predicting $\mathrm{BC}$ prognosis based on a comprehensive analysis. However, the mechanisms of GRP94 in BC need to be further verified both in vivo and in vitro.

\section{Acknowledgments}

Funding: This work was financially supported by grants from the National Basic Research Program of China (973 Program) (No. 2015CB553904), the CAMS Innovation Fund for Medical Sciences (CIFMS) (No. 2016-I2M-1-001, 2019-I2M-1-003), the National Natural Science Foundation of China (No. 81572842, 81672459, 81872280), the Nonprofit Central Research Institute Fund of Chinese Academy of Medical Sciences (No. 2017PT31029), the Open Issue of State Key Laboratory of Molecular Oncology (No. SKLKF-2017-16), and the Independent Issue of State Key Laboratory of Molecular Oncology (No. SKL-2017-16).

\section{Footnote}

Peer Review File: Available at http://dx.doi.org/10.21037/tcr$20-1853$

Data Sharing Statement: Available at http://dx.doi. org/10.21037/tcr-20-1853

Conflicts of Interest: All authors have completed the ICMJE uniform disclosure form (available at http://dx.doi. org/10.21037/tcr-20-1853). The authors have no conflicts of interest to declare.

Ethical Statement: The authors are accountable for all aspects of the work in ensuring that questions related to the accuracy or integrity of any part of the work are appropriately investigated and resolved. Part of our study was investigated using public databases including Oncomine, cBioPortal and Kaplan Meier plotter databases. The BC TMAs were purchased from Shanghai Outdo Biotech Co., Ltd. All procedures performed in studies were in accordance with the declaration of Helsinki (as revised in 2013). This study was approved by the Ethics Committee of Cancer Institute and Hospital, Chinese Academy of Medical Sciences (Approval No.16-038/1117), and all of the participants had been giving informed consent before the study.

Open Access Statement: This is an Open Access article distributed in accordance with the Creative Commons Attribution-NonCommercial-NoDerivs 4.0 International License (CC BY-NC-ND 4.0), which permits the noncommercial replication and distribution of the article with the strict proviso that no changes or edits are made and the original work is properly cited (including links to both the formal publication through the relevant DOI and the license). See: https://creativecommons.org/licenses/by-nc-nd/4.0/.

\section{References}

1. Jia $\mathrm{M}$, Zheng R, Zhang S, et al. Female breast cancer 
incidence and mortality in 2011, China. J Thorac Dis 2015;7:1221-6.

2. Li T, Mello-Thoms C, Brennan PC. Descriptive epidemiology of breast cancer in China: incidence, mortality, survival and prevalence. Breast Cancer Res Treat 2016;159:395-406.

3. Lee AS, Delegeane A, Scharff D. Highly conserved glucose-regulated protein in hamster and chicken cells: preliminary characterization of its cDNA clone. Proc Natl Acad Sci U S A 1981;78:4922-5.

4. Hoter A, El-Sabban M, Naim H. The HSP90 Family: Structure, Regulation, Function, and Implications in Health and Disease. Int J Mol Sci 2018;19:2560.

5. Yang Z, Zhuang L, Szatmary P, et al. Upregulation of Heat Shock Proteins (HSPA12A, HSP90B1, HSPA4, HSPA5 and HSPA6) in Tumour Tissues Is Associated with Poor Outcomes from HBV-Related Early-Stage Hepatocellular Carcinoma. Int J Med Sci 2015;12:256-63.

6. Xu Y, Chen Z, Zhang G, et al. HSP90B1 overexpression predicts poor prognosis in NSCLC patients. Tumor Biology 2016;37:14321-8.

7. Ji JJ, Fan J. Discovering myeloid cell heterogeneity in the lung by means of next generation sequencing. Mil Med Res 2019;6:33.

8. Takahashi H, Wang JP, Zheng HC, et al. Overexpression of GRP78 and GRP94 is involved in colorectal carcinogenesis. Histol Histopathol 2011;26:663-71.

9. Fu Z, Deng H, Wang X, et al. Involvement of ER- $\alpha 36$ in the malignant growth of gastric carcinoma cells is associated with GRP94 overexpression. Histopathology 2013;63:325-33.

10. Langer R, Feith $M$, Siewert JR, et al. Expression and clinical significance of glucose regulated proteins GRP78 (BiP) and GRP94 (GP96) in human adenocarcinomas of the esophagus. Bmc Cancer 2008;8:70.

11. Buc Calderon P, Sennesael A, Glorieux C. Glucoseregulated protein of $94 \mathrm{kDa}$ contributes to the development of an aggressive phenotype in breast cancer cells. Biomed Pharmacother 2018;105:115-20.

12. Yin N, Liu Y, Khoor A, et al. Protein Kinase $\mathrm{C} t$ and Wnt/ $\beta$-Catenin Signaling: Alternative Pathways to Kras/ Trp53-Driven Lung Adenocarcinoma. Cancer Cell 2019;36:156-67.e7.

13. Curtis C, Shah SP, Chin SF, et al. The genomic and transcriptomic architecture of 2,000 breast tumours reveals novel subgroups. Nature 2012;486:346-52.

14. Györffy B, Lanczky A, Eklund AC, et al. An online survival analysis tool to rapidly assess the effect of 22,277 genes on breast cancer prognosis using microarray data of 1,809 patients. Breast Cancer Res Treat 2010;123:725-31.

15. Marzec M, Eletto D, Argon Y. GRP94: An HSP90-like protein specialized for protein folding and quality control in the endoplasmic reticulum. Biochim Biophys Acta 2012;1823:774-87.

16. Yang Y, Li Z. Roles of heat shock protein gp96 in the ER quality control: redundant or unique function? Mol Cells 2005;20:173-82.

17. Chen Y, Chen C, Ma C, et al. Expression of heat-shock protein gp96 in gallbladder cancer and its prognostic clinical significance. Int J Clin Exp Pathol 2015;8:1946-53.

18. Sabbatino F, Favoino E, Wang Y, et al. Grp94-specific monoclonal antibody to counteract BRAF inhibitor resistance in BRAFV600E melanoma. J Transl Med 2015;13:K12.

19. Zhang LY, Zhang XC, Wang LD, et al. Increased expression of GRP94 protein is associated with decreased sensitivity to adriamycin in ovarian carcinoma cell lines. Clin Exp Obstet Gynecol 2008;35:257-63.

20. Chhabra S, Jain S, Wallace C, et al. High expression of endoplasmic reticulum chaperone grp94 is a novel molecular hallmark of malignant plasma cells in multiple myeloma. J Hematol Oncol 2015;8:77.

21. Shinagawa N, Yamazaki K, Tamura Y, et al. Immunotherapy with dendritic cells pulsed with tumorderived gp96 against murine lung cancer is effective through immune response of CD8+ cytotoxic $\mathrm{T}$ lymphocytes and natural killer cells. Cancer Immunol Immunother 2008;57:165-74.

22. Morales C, Rachidi S, Hong F, et al. Immune chaperone gp96 drives the contributions of macrophages to inflammatory colon tumorigenesis. Cancer Res 2014;74:446-59.

23. Dai YJ, Qiu YB, Jiang R, et al. Concomitant high expression of ERalpha36, GRP78 and GRP94 is associated with aggressive papillary thyroid cancer behavior. Cell Oncol (Dordr) 2018;41:269-82.

24. Rachidi S, Sun S, Wu BX, et al. Endoplasmic reticulum heat shock protein gp96 maintains liver homeostasis and promotes hepatocellular carcinogenesis. J Hepatol 2015;62:879-88.

25. Zhang $X$, Zhang L, Wang S, et al. Decreased functional expression of Grp78 and Grp94 inhibits proliferation and attenuates apoptosis in a human gastric cancer cell line in vitro. Oncol Lett 2015;9:1181-6.

26. Shen M, Jiang YZ, Wei Y, et al. Tinagl1 Suppresses Triple-Negative Breast Cancer Progression and Metastasis 
by Simultaneously Inhibiting Integrin/FAK and EGFR Signaling. Cancer Cell 2019;35:64-80.e7.

27. Lu L, Dong J, Wang L, et al. Activation of STAT3 and $\mathrm{Bcl}-2$ and reduction of reactive oxygen species (ROS) promote radioresistance in breast cancer and overcome of radioresistance with niclosamide. Oncogene 2018;37:5292-304.

28. Astvatsaturyan K, Yue Y, Walts AE, et al. Androgen receptor positive triple negative breast cancer: Clinicopathologic, prognostic, and predictive features.

Cite this article as: Wang T, Yang L, Li C, Wang J, Zhang J, Zhou Y, Sun F, Wang H, Ma F, Qian H. Comprehensive analysis reveals GRP94 is associated with worse prognosis of breast cancer. Transl Cancer Res 2021;10(1):298-309. doi: $10.21037 /$ tcr-20-1853
Plos One 2018;13:e0197827.

29. Zhang M, Han N, Jiang Y, et al. EGFR confers radioresistance in human oropharyngeal carcinoma by activating endoplasmic reticulum stress signaling PERKeIF2alpha-GRP94 and IRE1alpha-XBP1-GRP78. Cancer Med 2018;7:6234-46.

30. Feng C, Wu B, Fan H, et al. NF-kappaB-induced gp96 up-regulation promotes hepatocyte growth, cell cycle progression and transition. Wei Sheng Wu Xue Bao 2014;54:1212-20. 